\title{
A Study of Current Medication of Sinupret Drops in Chinese Pediatric Patients
}

Jianwen Shen ( $\square$ jianwen.shen@fdeent.org )

Fudan University Eye Ear Nose and Throat Hospital https://orcid.org/0000-0002-6562-3036

Jingchao Yan

Fudan University Eye Ear Nose and Throat Hospital

Research article

Keywords: Dosage, pediatric patients, prescriptions, Sinupret drops

Posted Date: May 13th, 2020

DOI: https://doi.org/10.21203/rs.3.rs-28326/v1

License: (9) This work is licensed under a Creative Commons Attribution 4.0 International License. Read Full License 


\section{Abstract}

Background Sinupret drops is commonly used as agent dissolving nasal secretions. However, there is no specific regime of Sinupret drops for pediatric patients in China. Therefore, we investigate its current medication in Chinese pediatric patients to provide a recommendation of safe drug use.

Methods We collect 6,893 outpatient and emergency pediatric prescriptions of Sinupret drops (age $\leq 15$ ) and focused on the analysis of practical drug dosage and potential risks.

Results One-half of the adult dosage was mainly chosen for out-patient children, while the full adult dosage was much more used for pediatric patients in emergency. Its usage in low-age pediatric patients requires further evaluation and surveillance. The pharmaceutical reaction of ethanol in Sinupret drops with some cephalosporins during drug taking will lead to unexpected drug reactions.

Conclusion To prevent potential side effects, treatment with Sinupret drops as an adjunct for pediatric patients should draw more attention from their doctors and pharmacists. The age and weight of pediatric patients need to be considered as important parameters for drug administration of Sinupret drops. It is important to strengthen the education of medication, and much attention should be paid to the interval of drug administration.

\section{Background}

Sinupret drops contains extracts of five herbal medicines, including elder flowers, primrose flowers with calyx, common sorrel, European vervain herb, and gentian roots [1]. It has been shown to promote the hydration effect in organs, improve the oscillation frequency of mucosa cilia, and deliquate the body secretion by speeding up their decomposition. Studies in vitro and in vivo have proved the broad spectrum of Sinupret drops antiviral activity and also show good anti-inflammatory, anti-bronchospasm and immunomodulatory effects [2-4]. Sinupret has been used in Europe for decades and more recently in the United States [5-6], and also in China [7], and is recommended as a Supplementary Specimen in Pharmacological Treatment of acute and chronic sinusitis [8].

As liquid drug, Sinupret drops can be taken together with fruit juice, and is thus much convenient in medication for children. Now, it has been widely used as a reagent dissolving secretions for pediatric patients of ENT departments in China. Till now, there is no regime specific for pediatric patients in the drug manual yet, and there is also no adjusted commercial form of Sinupret drops specialized for children in China. Nevertheless, this drug contains a high level of ethanol (19\%), which may introduce unexpected side-effect in the medication for children. Therefore, this study conducted data analysis on the practical use of Sinupret drops in pediatric patients in our EYE \& ENT hospital affiliated to Xxx University in Shanghai, the top 1 of the corresponding special hospitals in China, aiming to provide reference for clinical use and safety evaluation.

\section{Methods}




\section{Study design}

This study was conducted in one of the largest Eye, Ear, Nose and Throat Hospital hospitals in China. Collectively, 6893 pediatric prescriptions (outpatient and emergency) of Sinupret drops and relative adverse drug events were analyzed in the first half year of 2019. The prescriptions information includes the age, gender, diagnosis, drug dosage, combined medication and medication duration of each patient.

\section{Procedure}

The prescriptions of Sinupret drops were evaluated in terms of dosage, indications, drug interactions and course of treatment. The dosing and indications was audited by comparing with local and international recommendation guidelines. All the electronic prescription were individually reviewed by two clinical Pharmacists, and those irrational ones in first review were further confirmed by a third senior pharmacist.

\section{Data Analyses}

Data were checked for completeness before actual analysis. Descriptive analysis, such as means and percentages, was conducted in this study. Excel statistical software package (2016) was used for statistical analyses.

\section{Results And Discussion}

In our hospital, most of Sinupret drops-containing prescriptions (6,893 in 7,421 prescriptions) were applied for pediatric patients aged 15 or below, among them, 4158 males and 2735 females. The average age of analyzed patients was $5.74 \pm 2.20$ years old. Pediatric patients aged between 4 and 6 account for a much high proportion (Fig. 1), indicating that the users of Sinupret in our hospital are mainly children at low age. Considering that $19 \%$ of alcohol in Sinupret and its potential consequent adverse drug reaction, it should be cautious for pediatric patients when using Sinupret drops. Therefore, we conducted an indepth investigation of these 6,893 prescriptions, and discussed the practical use of this drug in our hospital.

\section{Dosage Analysis Of Sinupret Drops In Pediatric Patients}

According to the drug manual, the valid dosage of Sinupret drops is $18 \mathrm{ml}$ daily. In practice, they were individually determined by every doctor's own decision in each case. We noticed that the actual dosage is largely independent of pediatric patients' age (15 or below). The majority of Sinupret drops dosage was $9 \mathrm{ml}(1 / 2$ of full dosage) or $6 \mathrm{ml}$ ( $1 / 3$ of full dosage) daily, with the minority as $12 \mathrm{ml}$ ( $2 / 3$ of full dosage) and $18 \mathrm{ml}$ (full dosage) daily. The number of other dosing regiments was almost negligible (Fig. 2). 
We wish to make it clear whether there is any correlation of disease urgency with the choice of Sinupret dosage by distinct doctors. Therefore, dosage analysis was carried out on the emergency prescriptions and the outpatient prescriptions. As shown in Table 1, the proportion of prescriptions with full dosage of Sinupret were indeed much higher in emergency cases (32.7\%). In comparison, we failed to find such dominance in the outpatient prescriptions (13.1\%). We further took the disease severity into consideration and selected the outpatient prescriptions with no less than 2 diagnoses. Intriguingly, in these outpatient cases, doctors selected a variety of distinct Sinupret dosage probably resulting from their experiences and preferences.

Table 1

Dosage of Sinupret drops in our emergency and outpatient prescriptions.

\begin{tabular}{|lllll|}
\hline & $\begin{array}{l}1 / 3 \text { dosage } \\
(\%)\end{array}$ & $\begin{array}{l}1 / 2 \text { dosage } \\
(\%)\end{array}$ & $\begin{array}{l}\text { Full dosage } \\
(\%)\end{array}$ & Others (\%) \\
\hline Emergency prescriptions (223) & 15.7 & 25.6 & 32.7 & 25.0 \\
\hline Outpatient prescriptions (6670) & 16.4 & 52.6 & 13.1 & 17.9 \\
\hline $\begin{array}{l}\text { Outpatient prescriptions } \\
\begin{array}{l}\text { (no less than 2 diagnoses) } \\
(874)\end{array}\end{array}$ & 11.2 & 33.5 & 9.2 & 46.1 \\
\hline
\end{tabular}

As a result, we grouped our doctors into three categories according to their Sinupret-containing prescription number in half a year: (1) Frequent (more than 50 prescriptions), (2) Median (between 10 and 50 prescriptions), and (3) Infrequent (less than 10 prescriptions) (Fig. 3). We found that doctors in category (1) are more uniform in their dosage preference and more inclined to use $1 / 2$ of full dosage ( $9 \mathrm{ml}$ daily), which may be a relatively safe and effective dosage. Doctors in category (2) do not have such common preference in Sinupret dosage, which more likely is a result of their own judgment and dosage preference. Doctors who prescribed fewer than 10 prescriptions were more inclined to use higher dosage (more than $1 / 2$ of dull dosage), with less concern to reducing the drug dosage in pediatric patients, which may result from their unfamiliarity with the potential drug risk.

Obviously, the choice of halving the full dosage is much dominant in our hospital probably due to its convenience and the conventional thinking of half dose reduction in children. Nevertheless, such fixation in Sinupret drops dosage may not be appropriate for younger pediatric patients, and further dosage adjustment based on the age (especially at age 3 and below) should be performed for flexibility [9]. Actually, during the study period, we noticed a case of nausea, vomiting, diarrhea and rash caused by Sinupret drops (20-month-old baby, $12 \mathrm{~kg}, 3 \mathrm{ml}$ tid, po) collected from the adverse drug reaction monitoring and reporting system in our hospital. Although doctors have reduced the dose, adverse reactions are still occurring.

Moreover, even for the same age group of pediatric patients, other consideration still needs to be taken, such as the child's weight or the severity of the disease. Safe and effective medications according to age 
are proposed in several Sinupret drops randomized controlled clinical trials in China [10-13]. For instance, it was reported that the dosage of $1 \mathrm{ml}$ tid has been used for children under 1-year-old [13], the dosage of $4 \mathrm{ml}$ tid for children between 1 and 6 years old, and $6 \mathrm{ml}$ tid for children between 7 and 12 years old [11]. In conclusion, the dosage of Sinupret drops for low-age pediatric patients in our hospital can be further refined by the improvement of clinical measurement.

\section{Off-label administration of Sinupret drops in pediatric patients in our hospital}

In addition to acute and chronic rhinosinusitis indicated in the manual of Sinupret drops, other symptoms such as secretory otitis media, adenoid hypertrophy, rhinitis, etc. are also treated by using Sinupret drops (Table 2). These diseases are clearly associated with sinusitis, and the cases of children with chronic sinusitis accompanied by adenoid hypertrophy or secretory otitis media are very common in previous reports [14-15]. If pediatric patients suffer from adenoid hypertrophy for long time, it will cause serious obstruction of eustachian tube, which consequently change the microbial environment of nasopharyngeal cavity and reduce air flow in respiration. It will also be accompanied by otitis media and other related inflammation if the disease deteriorates. As a result, it is reasonable for the off-label application of Sinupret drops in upper respiratory tract diseases other than sinusitis if considering its antiinflammatory, anti-bronchospasm and immunomodulatory effects [2-3].

Table 2

Diagnoses of patients at age 15 or below in actual use of Sinupret drops.

\begin{tabular}{|lll|}
\hline Diagnoses & Prescription number & Proportion (\%) \\
\hline Sinusitis or rhinitis without otitis media & 2635 & 38.2 \\
\hline Secretory otitis media without sinusitis & 2433 & 35.3 \\
\hline Sinusitis with otitis media & 23 & 0.3 \\
\hline Adenoid hypertrophy/proliferative hypertrophy/hyperplasia & 1170 & 17 \\
\hline Others & 632 & 9.2 \\
\hline Total & 6893 & 100 \\
\hline
\end{tabular}

\section{The Combined Medication Of Sinupret Drops}

Sinupret drops are often used as an adjuvant drug. In our collected data, 6384 prescriptions (about $92.6 \%$ ) of Sinupret drops were used in combination with other drugs, more often in the form of 3 drugcombination, rather than its single use (Table 3 ). These combined drugs include unilateral drugs such as hormones (5714 prescriptions), antimicrobial drugs (3141 prescriptions) and anti-allergy drugs (1671 prescriptions), as well as compound drugs including compound preparation and Chinese patent drugs, 
which collectively comprise 102 kinds of distinct drugs (Table 4). Next, we discussed the combined application of Sinupret drops with different groups of unilateral drugs below.

Table 3

The single or combined medication of Sinupret drops in our hospital.

\begin{tabular}{|lll|}
\hline Sinupret drops medication & Prescription number & Proportion (\%) \\
\hline in single use & 509 & 7.4 \\
\hline 2 drug-combination & 1748 & 25.4 \\
\hline 3 drug-combination & 2638 & 38.3 \\
\hline 4 drug-combination & 1609 & 23.3 \\
\hline 5 drug-combination & 389 & 5.6 \\
\hline Total & 6893 & 100.0 \\
\hline
\end{tabular}


Table 4

The drug types used in combination with Sinupret drops and their common drugs. The corresponding prescription numbers are included in brackets

\begin{tabular}{|c|c|}
\hline Drug types & $\begin{array}{l}\text { Examples of frequently used drugs in the prescriptions of } \\
\text { Sinupret drops }\end{array}$ \\
\hline \multirow[t]{2}{*}{ Hormones (5714) } & Mometasone furoate nasal spray (3392) \\
\hline & Budesonide nasal spray (44) \\
\hline \multirow{7}{*}{$\begin{array}{l}\text { Antimicrobials (systemic) } \\
\text { (1147) }\end{array}$} & Azithromycin for suspension/Tablets $(523 / 14)$ \\
\hline & Cefaclor for Suspension/Capsules (462/33) \\
\hline & Cefprozil Dispersible Tablets (62) \\
\hline & Clarithromycin Tablets (25) \\
\hline & Cefalexin Sustained-release Capsules (14) \\
\hline & Cefdinir Capsules (11) \\
\hline & Metronidazole and Sodium Chloride Injection (1) \\
\hline \multirow[t]{2}{*}{ Antimicrobials (topical) (1994) } & Levofloxacin Hydrochloride Ear Drops (42) \\
\hline & Ofloxacin Ear Drops (118) \\
\hline \multirow{3}{*}{$\begin{array}{l}\text { Anti-allergy drugs (systemic) } \\
\text { (1092) }\end{array}$} & Cetirizine Hydrochloride Drops/Tablets $(473 / 17)$ \\
\hline & Montelukast Sodium Chewable Tablets (449) \\
\hline & Loratadine Syrup (132) \\
\hline \multirow[t]{3}{*}{ Anti-allergy drugs (topical) (579) } & Levocarbastine nasal spray (119) \\
\hline & Azostatin nasal spray (50) \\
\hline & Ketotifen solution (15) \\
\hline
\end{tabular}

\section{(1) The Combined Medication Of Sinupret Drops With Hormones}

Nasal hormones are the hormones with which Sinupret drops is most frequently used in combination, such as mometasone furoate nasal spray (3392 prescriptions) (Table 4). Their combination was applied against the chronic inflammation of the nasal cavity. Sinupret drops can disrupt the biomembrane of adenoid body, facilitating to exert hormone activities in anti-inflammatory, anti-edema and anti-allergic reactions. Meanwhile, Sinupret drops can also attenuate the atrophic and destructive changes of the ciliated epithelium, thereby reducing the adverse reaction of nasal bleeding caused by nasal hormones. ${ }^{16}$ These are the reasonable basis for this type of medication. 


\section{(2) The Combined Medication Of Sinupret Drops With Antimicrobials}

In this study, systemic and topical antimicrobial agents were used in 57\% prescriptions (1147 in 1994), respectively (Table 4), which shows a relatively high usage ratio. Azithromycin (523 prescriptions) and Cefaclor (462 prescriptions) were the most frequently used antibiotics when combined with Sinupret drops (Table 4). It has been reported that the combination of Sinupret with antimicrobial agents can effectively relieve the symptoms of acute sinusitis [1]. Therefore, it inferred that Sinupret can reduces the use of antibiotics in such diseases. On the other hand, since Sinupret drops contain 19\% of ethanol, its use in combination with some cephalosporin antibiotics and metronidazole will lead to dithiolam-like reactions $[17,18]$. Actually, one hospital in Jiangsu Province has banned the use of Sinupret and cephalosporin in the software system of rational drug use [19]. Therefore, medical education for both doctors and patients should be conducted that Sinupret drops needs to be taken with other cephalosporins at intervals as long as possible.

\section{(3) The Combined Medication Of Sinupret Drops With Anti- allergen}

Among the 1092 prescriptions of combined medication with anti-allergic drugs, near half (490) contain Cetirizine (Table 4) due to allergic rhinitis. Cetirizine is a second-generation anti-allergic drug and is considered relatively safe and effective in reducing symptoms associated with allergic rhinitis in children [20]. Notably, alcohol can activate the potential adverse reactions of Cetirizine and increase the risk of excessive sedation. Sinupret drops comprise $19 \%$ of alcohol, and their coexistence in the same prescription may bring unexpected drug risk, which should be informed to drug dispensers, usually those babysitting parents at home, about the medication interval of these drugs.

\section{The Duration Of Sinupret Drops Treatment}

In our hospital, the duration of Sinupret drops treatment is mainly within 15-30 days (Table 5). In comparison, the treatment cycle of chronic sinusitis and secretory otitis media is much longer, usually more than 12 weeks, and thus pediatric patients may repeatedly visit the hospital. As mentioned above (Fig. 3), medication education should be strengthened to all the doctors so as to avoid medication that may lead to high-risk pharmaceutical reaction and consequence. 
Table 5

Treatment duration of Sinupret drops in our hospital.

\begin{tabular}{|lll|}
\hline Treatment duration (days) & Prescription number & Proportion (\%) \\
\hline $3-7$ & 1412 & 20.48 \\
\hline $8-14$ & 2533 & 36.75 \\
\hline $15-30$ & 2844 & 41.26 \\
\hline $31-60$ & 101 & 1.47 \\
\hline $61-90$ & 2 & 0.03 \\
\hline 150 & 1 & 0.01 \\
\hline Total & 6893 & 100.0 \\
\hline
\end{tabular}

\section{Other Potential Risk Of Sinupret Drops}

We noticed that there are 5,050 prescriptions in which Sinupret drops were used in combination with topical drops or sprays. Practically, pharmacists in our hospital inform the patients of the drug usage orally and in print. Additionally, the correct use of Sinupret drops is also publicized on the popular platform such as WeChat Subscription. However, Sinupret drops has still been misused as ear drops or nose drops in our collected cases. Wrong drug delivery is largely responsible for the medication error [21]. As a result, it is strongly recommended to increase warning signs and to strengthen medication education for drug dispensers of pediatric patients.

\section{Strengths And Limitations}

Only one case of Sinupret drops' adverse drug reactions (ADR) was reported in the voluntary reporting system in our hospital, and the incidence is much likely underestimated. We hope to strengthen the surveillance and collect more relevant data in the future. Such information would help us to further analyze whether the relationship between ADR and drug interactions is clinical significant.

\section{Conclusion}

For pediatric patients, their age and weight should be considered as important parameters for administration of Sinupret drops. Ethanol as an excipient of Sinupret drops may interact with some commonly used antibiotics and anti-allergic drugs. Therefore, it is necessary to strengthen drug education for the interval of medication. Moreover, since the dosage form is drops, patients need to be informed for the correct route of drug administration to avoid external use.

\section{Declarations}




\section{Ethics approval and consent to participate}

All procedures performed in this study involving human participants were in accordance with the ethical standards of the institutional research committee and with the 1964 Helsinki declaration and its later amendments or comparable ethical standards. The Ethics Committee of Shanghai Eye, Ear, Nose and Throat Hospital, Shanghai Fudan University, Shanghai, China, approved this study.

\section{Consent for publication}

Not applicable.

\section{Competing interests}

The authors declare that they have no competing interests.

\section{Funding}

None

\section{Authors' contributions}

Both authors contributed to the design and implementation of this research, the analysis of the results, and writing of the manuscript. Both authors approved the final version of the manuscript to be published.

\section{Acknowledgements}

The authors are grateful to Dr.Yan Zhu for his kind support.

\section{References}

1. Melzer J, Saller R, Schapowal A, et al. Systematic review of clinical data with BNO-101 (Sinupret) in the treatment of sinusitis. Forsch Komplementmed. 2006;13(2):78-87. https://doi.org/10.1159/000091969.

2. Glatthaar-Saalmuller B, Rauchhaus U, Rode S, et al. Antiviral activity in vitro of two preparations of the herbal medicinal product Sinupret $(\mathrm{R})$ against viruses causing respiratory infections. Phytomedicine. 2011;19(1):1-7. https://doi.org/10.1016/j.phymed.2011.10.010.

3. Zhang S, Skinner D, Hicks SB, et al. Sinupret activates CFTR and TMEM16A-dependent transepithelial chloride transport and improves indicators of mucociliary clearance. PLoS One. 2014;9(8):e104090. https://doi.org/10.1371/journal.pone.0104090.

4. Cho DY, Skinner D, Mackey C, et al. Herbal dry extract BNO 1011 improves clinical and mucociliary parameters in a rabbit model of chronic rhinosinusitis.[J]. International forum of allergy rhinology. 2019;9(6):629-37. https://doi.org/10.1002/alr.22290. 
5. Palm J, Steiner I, Abramov-Sommariva D, et al. Assessment of efficacy and safety of the herbal medicinal product BNO 1016 in chronic rhinosinusitis. Rhinology. 2017;55: 142-51. https://doi.org/ 10.4193/Rhin16.103.

6. Jund $\mathrm{R}$, Mondigler $\mathrm{M}$, Steindl $\mathrm{H}$, et al. Clinical efficacy of a dry extract of five herbal drugs in acute viral rhinosinusitis. Rhinology. 2012;50(4):417-26. https://doi.org/10.4193/Rhino12.015.

7. Liu Z, Chen J, Cheng L. Chinese Society of Allergy and Chinese Society of Otorhinolaryngology-Head and Neck Surgery Guideline for Chronic Rhinosinusitis. Allergy Asthma Immunol Res. 2020;12(2):176-237. https://doi.org/10.4168/aair.2020.12.2.176.

8. Golusiński W. Recommendation for Sinupret as a supplementary specimen in pharmacological treatment of rhinosinusitis. Otolaryngologia polska. 2013;67(5):223-7. https://doi.org/10.1016/j.otpol.2013.08.001.

9. Son MJ, Kim YE, Song YI, et al. Herbal medicines for treating acute otitis media: A systematic review of randomised controlled trials. Complement ther med. 2017;35:133-9. https://doi.org/10.1016/j.ctim.2017.11.001.

10. Lin JH, Wang ZN, Ouyang JP. The curative effect of Sinupret on acute otitis media with middle ear effusion. Cent China Med J. 2009;33:80-4. http://en.cnki.com.cn/Article_en/CJFDTOTALHYXZ200902013.htm.

11. Yang JM, Meng XT,Yang X. Efficacy of Sinupret drops therapy in children with rhinosinusitis and its influence on inflammatory factors. Chinese Journal of General Practice. 2018;16:4586-4552. http://en.cnki.com.cn/Article_en/CJFDTotal-SYQY201804024.htm.

12. Liu ZJ, Zhang XL, Wang X. Efficacy and Safety of Olongma Drops Combined with Cefpodoxime Ester in the Treatment of Secretory Otitis Media in Infants Aged 8-12 Months. China Journal of Pharmaceutical Economics. 2019;14(9): 42-45. http://zywa.chinajournal.net.cn/WKD/WebPublication/paperDigest.aspx?paperID=698a1a3a-69e84a65-914d-86e0542abcec.

13. Shen J, Dang H. Observation on the Safety and Efficacy of Sinupret in the Treatment of Pediatric Sinusitis. China Pharmacy. 2006;17(12):930-2. http://en.cnki.com.cn/Article_en/CJFDTOTALZGYA200612024.htm.

14. Shen L, Xu YY, Lin ZT, et al. [Relationship between children's obstructive sleep apnea hypopnea syndrome and nasal diseases]. Chinese journal of otorhinolaryngology head neck surgery. 2013;48:507-10. http://www.wanfangdata.com.cn/details/detail.do? _type=perio\&id=zhebyhk201306015.

15. Lin DL, Wu CS, Tang $\mathrm{CH}$, et al. The safety and risk factors of revision adenoidectomy in children and adolescents: A nationwide retrospective population-based cohort study. Auris nasus larynx. 2018;45(6):1191-8. https://doi.org/10.1016/j.anl.2018.03.002.

16. Peric A, Kovacevic S, Gacesa D, et al. Efficacy and safety of combined treatment of acute rhinosinusitis by herbal medicinal product Sinupret and mometasone furoate nasal spray. ENT Updates. 2017;7(2):68-74. https://doi.org/10.2399/jmu.2017002003. 
17. Ren S, Cao Y, Zhang X, et al. Cephalosporin induced disulfiram-like reaction: a retrospective review of 78 cases. Int surg. 2014;99(2):142-6. https://doi.org/10.9738/INTSURG-D-13-00086.1.

18. Xu J, Cai HH, Liu B, et al. One case of disulphulam-like reaction in children caused by the interaction of olongma drops and cephalosporin. China Pharmacist. 2017;20(11):2035-6. http://www.cnki.com.cn/Article/CJFDTOTAL-ZYSG201711039.htm.

19. Chen $\mathrm{R}$, Gu BC, Gong YH. Application of rational drug use software system in the drug dispensing in outpatient department of our hospital. China Pharmacy. 2016;27(22):3091-4. http://www.en.cnki.com.cn/Article_en/CJFDTOTAL-ZGYA201622021.htm.

20. Phan H, Moeller ML, Nahata MC. Treatment of allergic rhinitis in infants and children: efficacy and safety of second-generation antihistamines and the leukotriene receptor antagonist montelukast. Drugs. 2009;69(18):2541-76. https://doi.org/10.2165/9884960-000000000-00000.

21. Jafarian K, Allameh Z, Memarzadeh M, et al. The Responsibility of Clinical Pharmacists for the Safety of Medication Use in Hospitalized Children: A Middle Eastern Experience. J Res Pharm Pract. 2019;8(2):83-91. https://doi.org/10.4103/jrpp.JRPP_19_66.

\section{Figures}

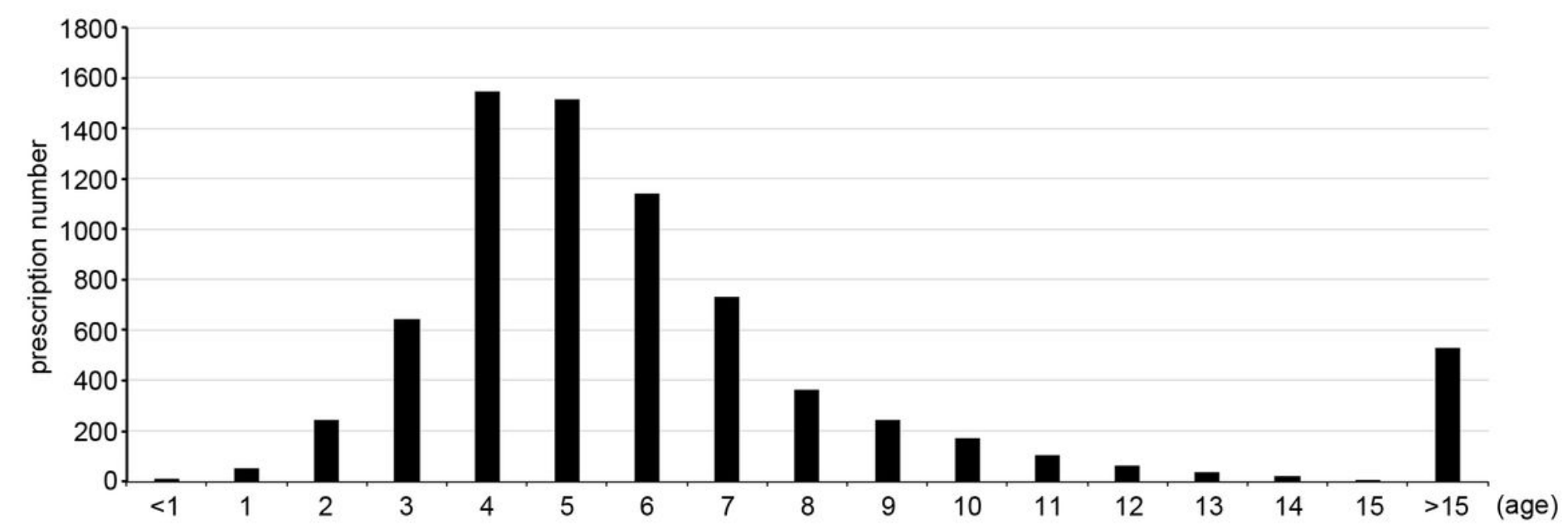

Figure 1

Distribution of pediatric patients' age and the prescription number of Sinupret drops in our hospital in the first half of 2019. 


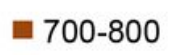

$=600-700$

$=500-600$

= 400-500

$=300-400$

$=200-300$

$=100-200$

$=0-100$

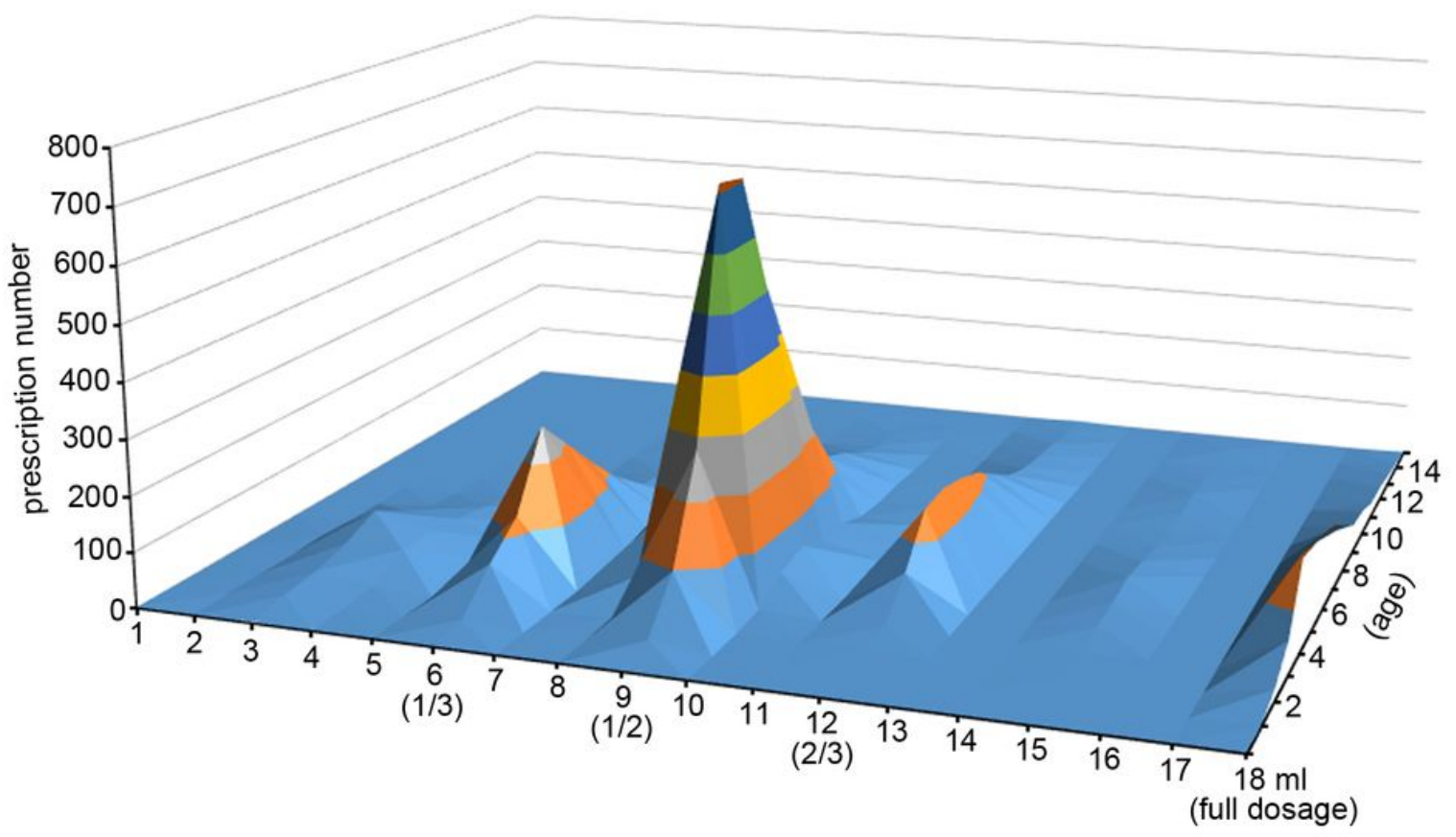

Figure 2

Administration of Sinupret drops in our hospital. The distribution of prescription number of Sinupret drops is shown in aspects to the drug dosage (18 $\mathrm{ml}$ daily as the full dosage for adults) and the age of pediatric patients. 


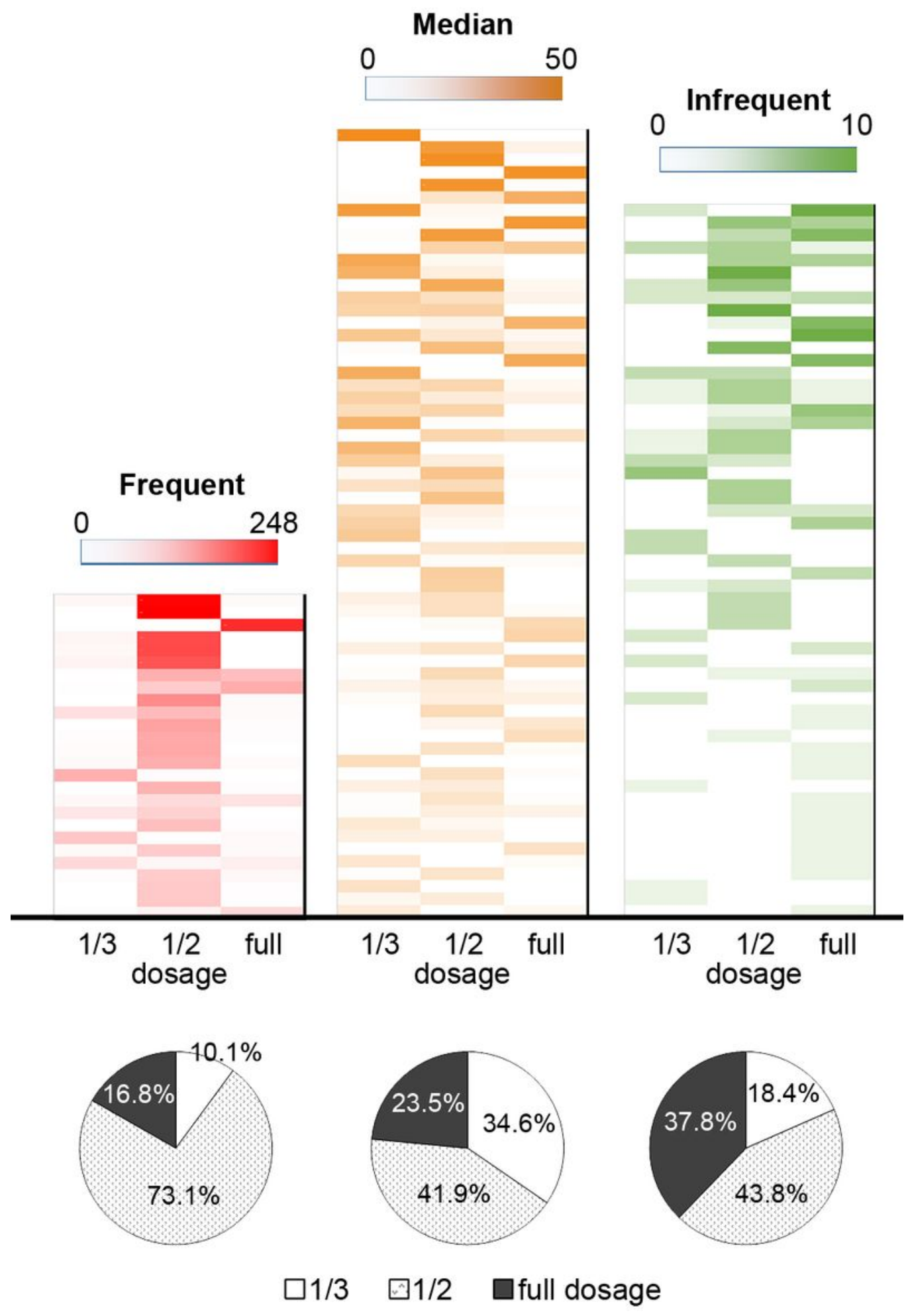

Figure 3

Correlation between doctors' prescription number of Sinupret drops and their selection of drug dosages. Doctors are grouped into three categories according to the number of their Sinupret-containing prescriptions in half a year: (1) Frequent (> 50 prescriptions), (2) Median (between 10 and 50 prescriptions), and (3) Infrequent (< 10 prescriptions). (Upper panel) Heat map of the prescription number. 
Each line represents one doctor, and different row represent the distinct dosage $(1 / 3,1 / 2$ and full dosage). Different colors correspond to the prescription numbers in three groups.

\section{Supplementary Files}

This is a list of supplementary files associated with this preprint. Click to download.

- renamedecece.pdf

- renamed15bcc.pdf 